\title{
Non-Collocated Displacement Sensing by Semiconductor Strain Gauges in Differentially Piezo-Driven Nanopositioners
}

\author{
Ali Bazaei, Member, IEEE, Mokrane Boudaoud, Massoud Hemmasian Ettefagh, Zhiyong Chen, Senior \\ Member, IEEE, Stephane Régnier
}

\begin{abstract}
We address design, implementation, and characterization of semiconductor strain gauges as stage displacement sensors with small footprint for piezo-driven nanopositioners in differential actuation mode. The strain gauges are not collocated with the stage of the nanopositioner. They are attached to piezo stack actuators in both longitudinal and transverse directions. We address two differential configurations for the displacement sensing. The first configuration uses all strain gauges and provides three output signals. Two of them are proportional to the length variations of the piezo actuators, which are considered as conventional non-differential sensing method. The last output in the first configuration is the first proposed differential sensor. In the second configuration, we propose an alternative differential sensor using only the longitudinal strain gauges and a simpler readout circuit. The results indicate that in the differential actuation mode, a differential sensing strategy provides much better accuracy than the conventional non-differential schemes. Using a laser interferometer as an independent collocated sensor for stage displacement, we obtained constant calibration factors both proposed differential sensors and characterized their sensing accuracies.
\end{abstract}

Index Terms-Displacement sensor, Semiconductor strain gauge, Piezoresistive sensor, Nanopositioner, Piezoelectric actuator, Differential actuation

\section{INTRODUCTION}

An efficient method to increase the range of motion in piezo-driven nanopositioners without sacrificing the mechanical bandwidth is to include actuators on opposing sides of the stage [1], [2]. This method provides bilateral displacement of the stage along the axis of excitation when the opposing actuators are driven by signals that are $180^{\circ}$ out of phase and biased by an identical positive voltage value. The method, which is known by several names such as dual-stack, push-pull, and differential actuation, has

A. Bazaei, M. Boudaoud, and S. Regnier are with the Institute for Intelligent Systems and Robotics (ISIR), Sorbonne University, Paris, France. M. H. Ettefagh is with Huazhong University of Science and Technology, China. A. Bazaei and Z. Chen are with the School of Electrical Engineering and Computing, University of Newcastle Australia, Callaghan, NSW 2308. Emails: ali.bazaei@newcastle.edu.au, boudaoud@isir.upmc.fr, massoud.ettefagh@gmail.com, zhiyong.chen@newcastle.edu.au, regnier@isir.upmc.fr.

An earlier version of this paper was presented at the 2019 IEEE SENSORS Conference and was published in its Proceedings: https://ieeexplore.ieee.org/document/8956836 been proven to improve the stroke and blocking force of the actuation mechanism compared to the conventional one-sided actuation scheme [3]. However, application of semiconductor strain gauges in displacement measurement has not been addressed for piezo-driven nanopositioners in the differential actuation mode.

A conventional method to measure displacement in flexure-based macro-nanopositioners is to utilize capacitive sensors [4] -7 . Such sensors are generally expensive, need additional space and parts for physical installation, and require bulky electronic modules to generate a real-time signal proportional to the target displacement. Other conventional displacement sensors, such as laser interferometers and Eddy current sensors also suffer from the foregoing issues $[8-10$. Piezoresistive strain gauge elements have also been used to measure displacement in nanopositioners 11$]-13$. To do this, one normally needs to glue multiple strain gauges to the suspension beams, configure them in a Wheatstone bridge, and use DC power supplies and amplifiers to generate an output signal 14 [16. In addition, installation of conventional gauges on suspension beams is usually manual and can introduce undesirable asymmetry in the nanopositioner structure (especially for those with smaller dimensions), which is normally designed symmetrical (to reduce cross-coupling) and is fabricated by high precision automated machines. Automatic installation of piezoresistive strain gauges on flexure hinges is also possible by micromachining processes, however, it requires to fabricate the nanopositioner from fragile materials such as silicon $[2$ or fabrication of the gauges on additional non-conductive flexible structures [17, 18.

In the differentially actuated mechanisms reported in 3 and [1] a commercial fiber optic instrument and straingauges were used for displacement measurement, respectively. However, the fiber-optic instrument needs bulky signal conditioning facilities and the strain-gauge sensing technique was not addressed in [1. To measure the stage displacement in the differentially driven nanopositioner reported in [2], piezoresistors were embedded into the side walls of the suspension beams in the monolithic silicon structure. However, most of the piezo-driven nanopositioners, including the prototype reported in this paper, are fabricated from commercial metals such as aluminum or iron alloys, where deposition of piezoresistive zones 
into the metallic structure is difficult. In addition, for nanopositioners with small dimensions such as the one proposed here, it is difficult to properly install commercial piezoresistive strain-gauges on the side walls of the suspension beams.

An alternative place to attach strain-gauges for stage displacement measurement in piezo-driven nanopositioners is the side walls of the PZT actuator. This method has been applied to many commercial PZT stack actuators with metal foil strain gauges. The sensing method has also been applied to piezo-driven nanopositioners, which are actuated from one side of the stage only [19], 20]. In 21], we presented an earlier version of this sensing method for piezo-driven nanopositioners in differential actuation mode. This performance is investigated with more details in this paper using semiconductor strain gauges, which are more sensitive than the metal foil counterparts. Compared to the differential sensor configuration presented in [21], we also propose an alternative differential sensing configuration, which is more compact and provides more displacement sensing accuracy. We also characterize noise and resolution of both proposed differential sensors and clarify dependency of the sensor noise level on the actuation signal magnitude.

\section{EXPERIMENTAL SETuP}

We designed a flexure-based nanopositioner symmetrically with respect to the $\mathrm{x}$ and $\mathrm{y}$ axes to incorporate dual piezo stacks for differential actuation of each axis. The monolithic structure of the nanopositioner was fabricated by wire-cut EDM (Electrical Discharge Machining) from 304 stainless steel as shown in Fig. 1] 22]. The square stage in the middle can be driven along $\mathrm{x}$ and/or $\mathrm{y}$ axes by piezo stack actuators via slender suspension beams. Figure 2 (a) shows the schematic diagram of $\mathrm{x}$-axis structure in the differential xy-nanopositioner along with an electrical excitation mechanism to differentially drive the piezoelectric actuators. We used a commercial dual channel voltage amplifier equipped with a phase inverter to drive the opposing piezo stacks by out-of-phase high voltage signals. The piezo stacks have the specifications reported in 23. (Model PK4DLP2). The external dimensions of the nanopositioner had to meet stringent limitations, especially in the vertical direction. The limitations are mainly due to restricted space available on a 6 -DoF positioning platform in a SEM (Scanning Electron Microscope) reported in 24]-27, where the proposed nanopositioner is to be installed. The nanopositioner after installation of all piezo stacks is shown in Fig. 1 (b). We have reported some attributes of the proposed nanopositioner and a few commercially available high-speed and/or low-profile x$y$ stages in Table II. This comparison reveals that the proposed nanopositioner is an exceptionally low-profile and high-speed flexure-based stage.

We also equipped the piezo stacks with semiconductor strain gauges, as indicated in Fig. 2(b). To obtain maximum sensor sensitivity, the gauges are unbacked (naked) and have been attached to the free side walls, where
Table I

SUMMARIZED SPECIFICATIONS OF THE PROPOSED NANOPOSITIONER COMPARED TO COMMERCIAL HIGH-SPEED AND LOW-PROFILE flexure-Based stages. The abbreviations "Commer." " "W", "L", "H", "Range", "Freq.", "Tech.", "SSG", and "CAP" refer to

"Commercial", "Width", "Length", "Height", "Travel Range", "Resonance Frequency", "Technology", "Silicon Strain Gauge", and "Capacitive", Respectively.

\begin{tabular}{ccccccc}
\hline $\begin{array}{c}\text { Commer. } \\
\text { Stage }\end{array}$ & $\begin{array}{c}\mathrm{W} \\
(\mathrm{mm})\end{array}$ & $\begin{array}{c}\mathrm{L} \\
(\mathrm{mm})\end{array}$ & $\begin{array}{c}\mathrm{H} \\
(\mathrm{mm})\end{array}$ & $\begin{array}{c}\text { Range } \\
(\mu \mathrm{m})\end{array}$ & $\begin{array}{c}\text { Freq. } \\
(\mathrm{kHz})\end{array}$ & $\begin{array}{c}\text { Sensor } \\
\text { Tech. }\end{array}$ \\
\hline$\overline{28}$ & 50.8 & 50.8 & 29.2 & 10 & 1.5 & SSG \\
\hline$\frac{29}{30}$ & 30 & 30 & 28 & 6 & 4.2 & CAP \\
\hline$\frac{31}{32}$ & 120.8 & 120.7 & 28.5 & 75 & $3 / 2$ & SSG \\
This work & 190 & 152.5 & 18.5 & 75 & $1.5 / 1$ & SSG \\
\hline
\end{tabular}

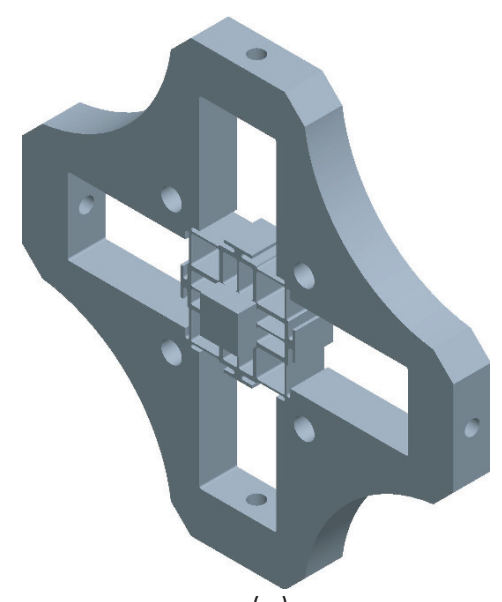

(a)

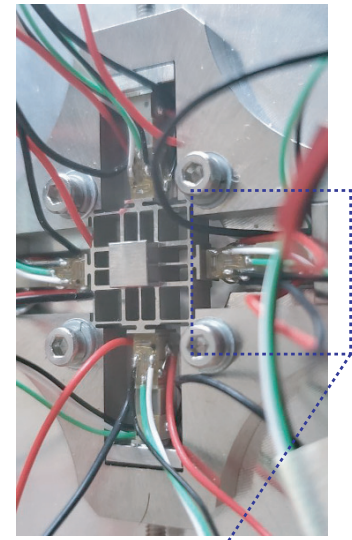

(b)

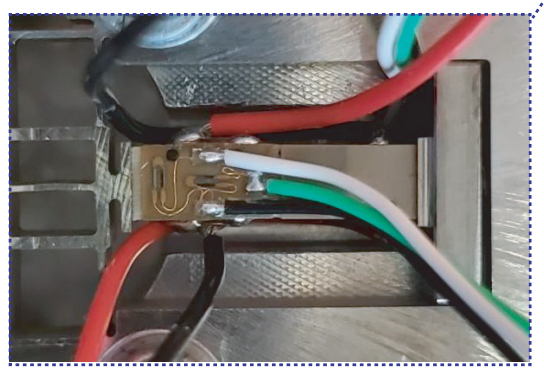

(c)

Figure 1. (a) CAD view of the monolithic structure of the nanopositioner before installation of actuators.(b) Completed nanopositioner after installation of piezo stack actuators. (c) Closeup view of the unbacked silicon strain gauges attached to top side wall of a piezo stack.

actuation electrodes do not present. On each free side wall of each actuator, two U-shaped silicon chip gauges have been attached, one in longitudinal direction and the other one in transverse direction as depicted in Figures 2 (b) and 1 (c). After attaching the gauges and bonding pads to the stacks and soldering the gauge wires, a thin silicone layer covers them to be more rugged for handling. For each actuator, the four gauges are matched and each one has a nominal resistance of $540 \Omega$, gauge factor of 140 , length of $1.53 \mathrm{~mm}$, width of $0.41 \mathrm{~mm}$, and thickness of $15.3 \mu \mathrm{m}$. The gauge part number is SS-060-033-500PU with more 
details reported in 33. Experiment shows that resistances of longitudinal and transverse gauges vary oppositely when the actuator is excited. Hence, each piezo stack is equipped with four strain gauges that allow to form a full Wheatstone bridge for each individual actuator. Since the piezoresistors are sensitive to temperature, we excited the bridges by a low DC voltage level and amplified each bridge output by an instrumentation amplifier (INAMP). For the first proposed sensor configuration, the schematic of readout circuit of $\mathrm{x}$-axis is as shown in Fig. 2 (c), where the gauge resistors in the top and bottom bridges are associated with the $x^{+}$and $x^{-}$actuators, respectively. We have included small potentiometers in each bridge for null shift compensation after application of $70 \mathrm{~V}$ electrical bias to the actuators. To further assist in zero balancing, we used switches to provide possibility of including the potentiometers in the left lower leg of the bridges. In this way, signals $v_{s x}^{+}$and $v_{s x}^{-}$in the readout circuit are proportional to variations in the length of $x^{+}$and $x^{-}$ actuators, respectively. The final INAMP in the readout circuit provides an output proportional to $v_{s x}^{+}-v_{s x}^{-}$with a smaller gain, which is considered as a differential output for stage displacement sensing. The nominal voltage gain of each INAMP is $1+\frac{50}{R_{g}}$ with the value of external gain resistance $R_{g}$ in $\mathrm{k} \Omega$.

A valid measurement of the stage displacement is also required for characterization of the piezoresistive sensors. One side of the middle stage is vertically extruded about $2 \mathrm{~mm}$ to provide direct displacement measurement by a laser interferometer 22$]$.

\section{A. A More Compact Differential Sensing Configuration}

We also consider an alternative differential sensing strategy that involves only one INAMP and smaller number of strain gauges. To measure stage displacement in this method, we apply only the longitudinal strain gauges in a full-bridge configuration and amplify the bridge output by an INAMP, as depicted in Fig. 3 In this configuration, the strain gauges in the transverse direction are left open circuited and not required in the readout circuit. The differential strategy in this case is achieved by the fact that the output voltage of the full-bridge is proportional to the difference between the variations of the longitudinal gauge resistance values on the $x^{+}$and $x^{-}$piezo actuators.

\section{SEnSOR BANDwidth}

Using the differential actuation method mentioned in Sec. II] we obtained the frequency responses from the input signal of $\mathrm{x}$-axis $\left(v_{i x}\right)$ to the stage displacement along $\mathrm{x}$-axis (measured by the laser interferometer) and the differential outputs $v_{s x}$ in the readout circuits of $\mathrm{x}$ axis, as shown in Fig. 4 Using the laser interferometer, the stage displacement in the differentially driven nanopositioner has a dominant resonance mode along $\mathrm{x}$ at $21.3 \mathrm{kHz}$. The dominant resonance frequency measured by the first proposed differential sensor in Fig. 2 (c) is

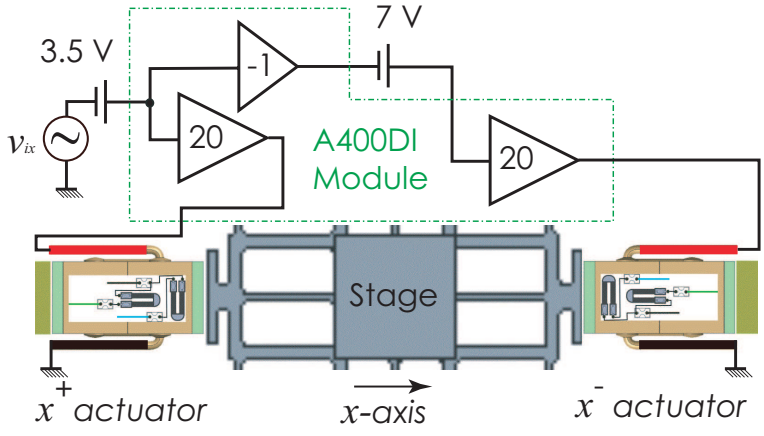

(a)

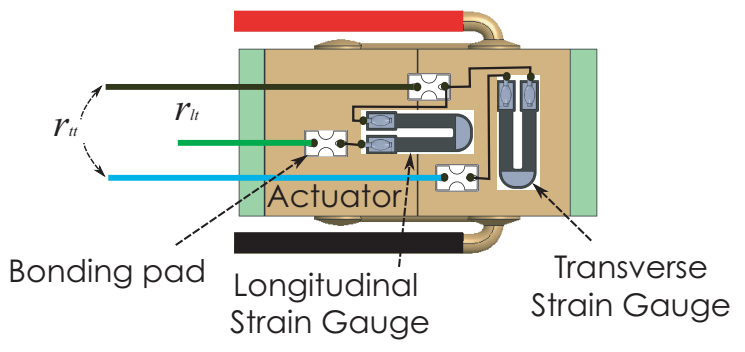

(b)

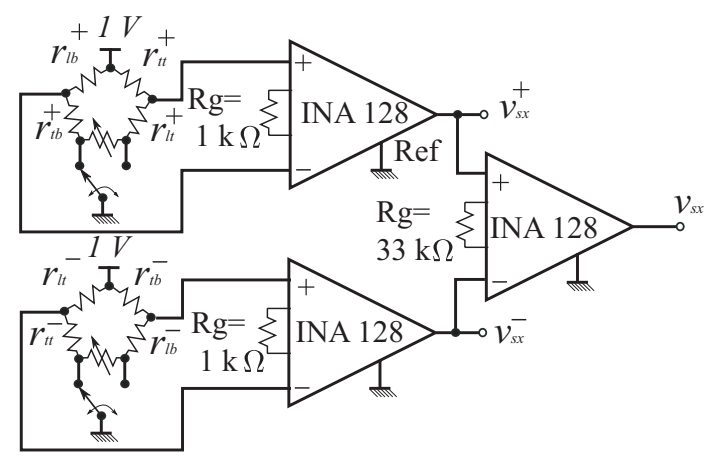

(c)

Figure 2. (a) Schematic of differential actuation of PZT stack actuators for $\mathrm{x}$-axis of the nanopositioner. The A400DI module is a dual channel voltage amplifier with a built in phase inverter. (b) Schematic of strain gauges attached to the top side of each actuator. Similar gauges are attached to the bottom side whose resistances are denoted by $r_{l b}$ and $r_{t b}$ along longitudinal and transverse directions, respectively. (c) PZR readout circuit of the first proposed configuration used for stage displacement sensing along $\mathrm{x}$-axis.

about $1 \mathrm{kHz}$ smaller than that of the stage displacement. Both displacement and differential sensor exhibit almost flat magnitude and phase responses up to $4 \mathrm{kHz}$. As frequency increases beyond this limit, the magnitude for stage displacement grows more rapidly than that of the differential sensor and so is the absolute difference between the two phase responses. Hence, the proposed differential sensor has an approximate bandwidth of $4 \mathrm{kHz}$ for direct measurement of the stage displacement. For the second sensor configuration described in Fig. 3 the measured frequency response to differential actuation is also included in Fig. 4. exhibiting almost the same phase and magnitude variation as those of the first sensor.

\section{Characterization of Sensor Outputs}

Considering the almost flat frequency responses of the stage displacement and the differential sensors, it is de- 


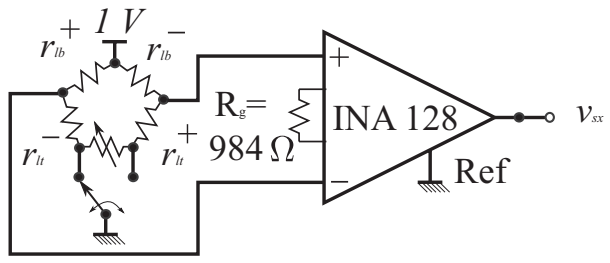

Figure 3. An alternative differential sensing approach using only the longitudinal strain gauges attached the driving piezo stacks. The symbols used for the gauge resistors are as described in Fig. 2 (b,c) with + and - superscripts indicating that the gauge is attached to $x^{+}$and $x^{-}$actuators, respectively.
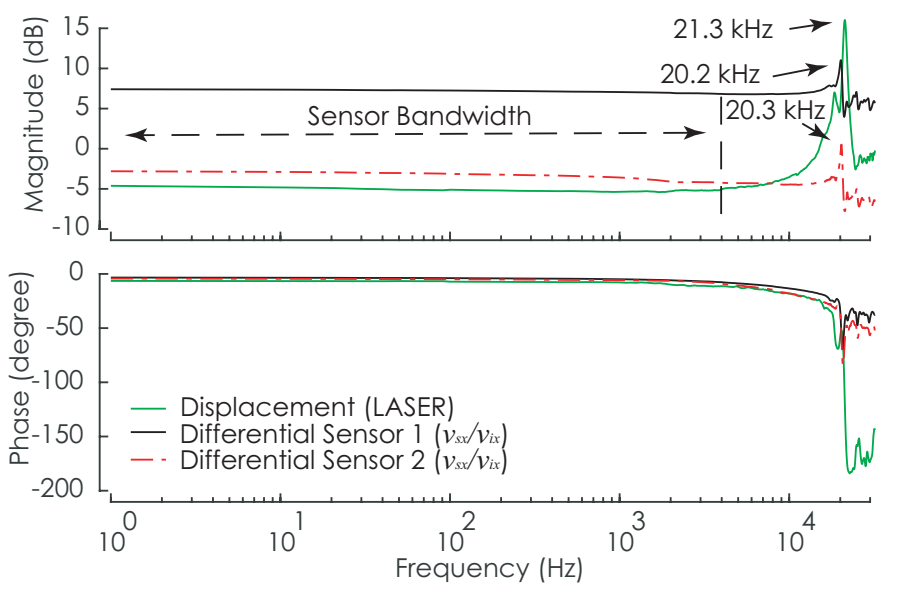

Figure 4. Frequency responses of the $\mathrm{x}$-axis in the differential actuation mode to the stage displacement (Laser) and the differential outputs of the first and the second proposed sensor configurations.

sirable to obtain constant calibration coefficients for each of the outputs in the readout circuits to measure stage displacement during the differential actuation.

Since the nanopositioner is lightly damped, we applied a large signal sinusoidal input to the differential actuation mechanism of the $\mathrm{x}$-axis at a frequency which is well within bandwidth of the proposed sensor. The sinusoidal waveform is the best choice for calibration as it has minor high frequency components and is less likely to excite resonance modes of the nanopositioner, which are out of the sensor bandwidth. Figure 5 (a) shows the stage displacement along x-axis (measured by the laser) while the $\mathrm{x}$-axis differential actuation mechanism is excited by a $400 \mathrm{~Hz}$ large signal sinusoid input. The figure also includes the actuation and sensor outputs of the first circuit configuration after multiplying them by scale factors and and adjusting their DC levels so that their extrema levels match those of the stage displacement. The legends also quote the scale factor values associated with each output in the first readout circuit, which are considered as the calibration coefficients of the proposed differential output $v_{s x}$ and the non-differential outputs $v_{s x}^{+}$and $v_{s x}^{-}$. The errors associated with each calibrated output shown in Fig. 5 (b) indicate that the proposed differential sensor provides a better accuracy than the non-differential ones during the differential actuation mode. It has also a better $3 \sigma$-resolution of $2.4 \mathrm{~nm}$ with respect to the non-differential
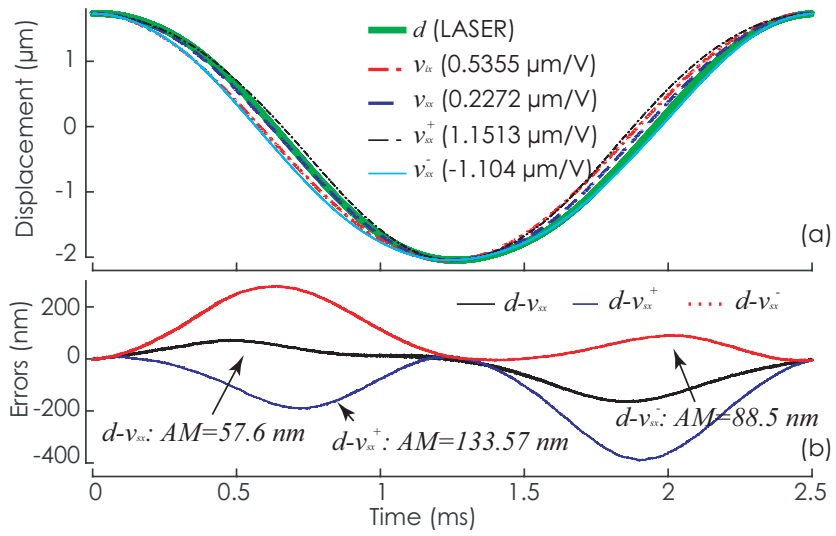

Figure 5. Calibration of the outputs in $\mathrm{x}$-axis readout circuit described in Fig. 2 (c) for stage displacement measurement, using a $400 \mathrm{~Hz}$ large signal sinusoidal excitation of $\mathrm{x}$-axis in the differential actuation mode. We have also indicated the average magnitude (AM) of error for each sensor in figure (b). The signals were collected by dSPACE with a sampling rate of $45 \mathrm{kHz}$, simultaneously. To increase the graph resolution, the data from many successive periods have been displayed in one period.

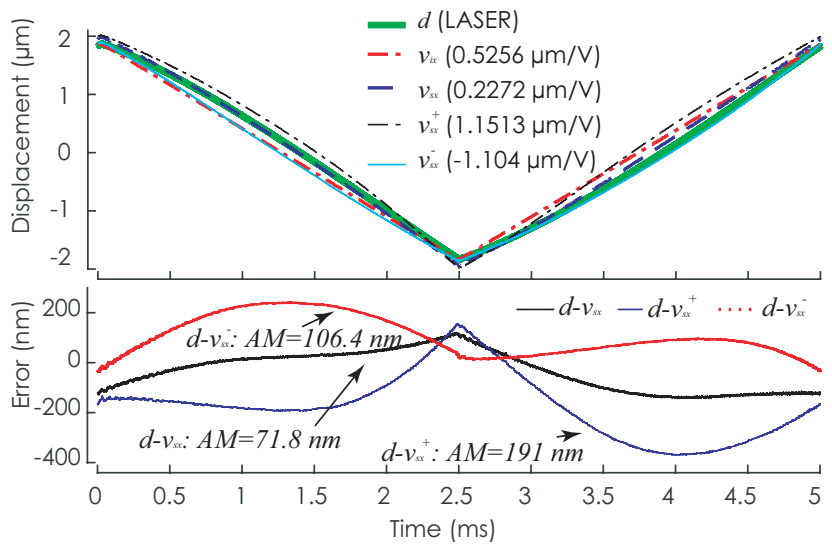

Figure 6. Examination of the differential sensor of x-axis in Fig. 2(c) in the differential actuation mode by a large signal $200 \mathrm{~Hz}$ triangular excitation using the calibration coefficients obtained in Fig. 5

outputs ( $7.7 \mathrm{~nm}$ and $5.3 \mathrm{~nm}$ for $v_{s x}^{+}$and $v_{s x}^{-}$, respectively).

We have also examined the outputs of the readout circuit in the differential actuation mode by a non-sinusoidal large signal actuation at a different frequency value within the sensing bandwidths using the calibration coefficients obtained earlier, as shown in Fig. 6. The average error magnitude for the differential sensor is around $1.96 \%$ of the $3.67 \mu \mathrm{m}$ displacement range, whilst those associated with $v_{s x}^{+}$and $v_{s x}^{-}$are $5.21 \%$ and $2.9 \%$, respectively.

\section{A. Characterization of the more compact differential sen- sor}

We also calibrated the sensor proposed in Fig. 3 by driving the stage along $\mathrm{x}$-axis in the differential actuation mode using a large signal sinusoidal excitation. The measured waveforms after applying the scale factors and the resulting sensor error are shown in Fig. 7 Note that the displacement sensing error in this case is smaller than 

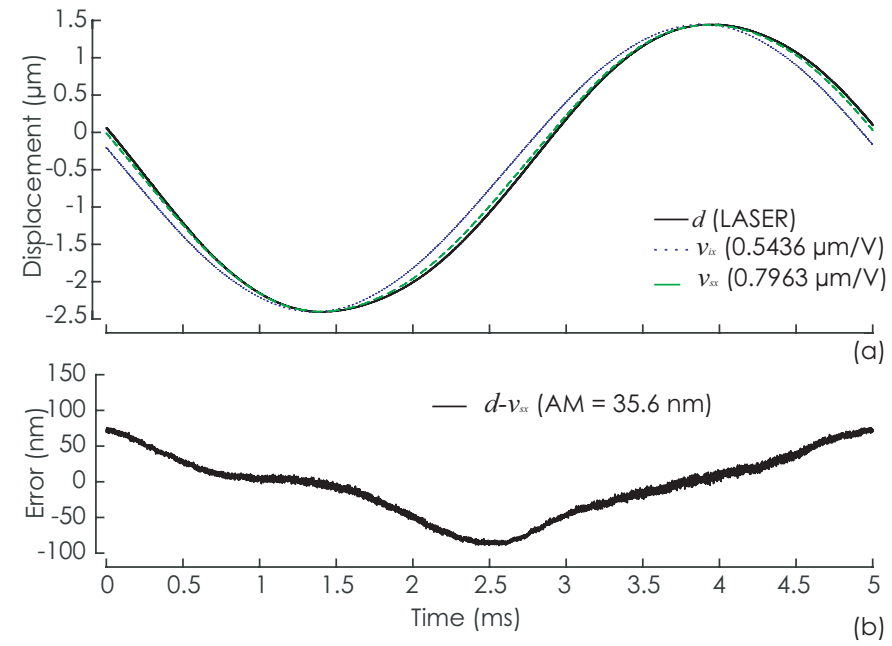

Figure 7. Results of the calibration test of the differential sensor in Fig. 3 using a $140 \mathrm{~V}_{p p}$ sinusoidal excitation of piezo stacks in the differential actuation mode.

that of the original differential sensor depicted in Fig. 5 (b) with $38 \%$ reduction in the average magnitude of the error (AM). Compared to the original differential sensor, the scale factor of the new differential sensor has now increased by a factor of 3.5, which means sensitivity of the differential sensor has decreased by the same factor. This decrease in sensitivity is mainly due to smaller voltage amplification gain and using only one full Wheatstone bridge in the readout circuit.

Considering that the value of gain resistor $R_{g}$ in the new version of the differential sensor is almost equal to the gain resistors in the first stage INAMPs in Fig. 2 (c), the voltage gain of the INAMP used in the new sensor is almost equal to the gain of each first stage INAMP in the readout circuit of the original sensor. The magnitudes of the scale factors associated with the outputs of the first stage INAMPs in Fig. 2 (c) $\left(v_{s x}^{+}\right.$and $\left.v_{s x}^{-}\right)$are around $1.128 \frac{\mu \mathrm{m}}{\mathrm{V}}$. Hence, the sensitivity of the full bridge used in the new differential sensor has been improved by a factor of 1.42 with respect to each individual full bridge used in the originally proposed sensor.

Using the constant scale factor of $0.7963 \frac{\mu \mathrm{m}}{\mathrm{V}}$ obtained during the calibration test, we also examined the new sensor by a large signal $100 \mathrm{~Hz}$ triangular waveform excitation. The measured signals and sensor error are shown in Fig. 8. Comparing with the validation test results of the original sensor reported in Fig. 66 the average magnitude of the displacement sensing is reduced $43 \%$ with the new configuration of differential sensing. Figure 9 shows the hysteresis curves associated with this validation test, where the sensor output closely follows the deviation of displacement from a straight line due to hysteresis nonlinearity of the piezo actuators. Hence, the new differential sensing strategy provides a more sensitive Wheatstone bridge and a more accurate displacement measurement.

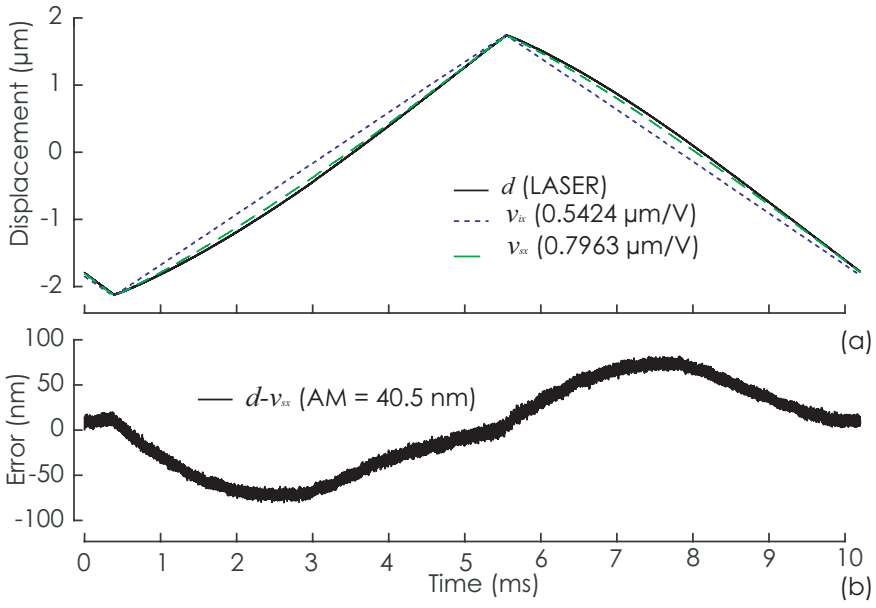

Figure 8. Results of the validation test of the differential sensor in Fig. 3 using a $140 \mathrm{~V}_{p p}$ triangular excitation of piezo stacks in the differential actuation mode.

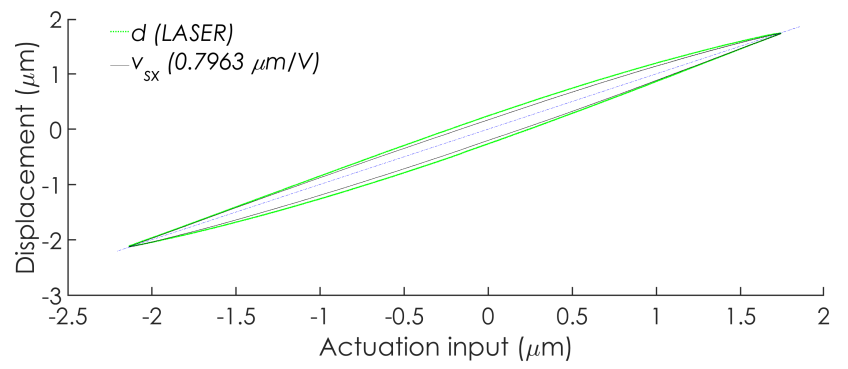

Figure 9. The hysteresis curves associated with the time domain signals in Fig. 8 (a).

\section{B. Sensor Noise and Resolution}

In this section, we characterize noise and resolution of the proposed differential displacement sensors during differential actuation mode. To clarify dependency of noise level on the actuation signal, we extracted the noise component from measured responses of the sensors while driving the nanopositioner by a large signal sinusoidal excitation. The noise extraction was carried out by calculating moving average of the measured time domain data and subtracting it from the measured data. We chose Savitzky-Golay filtering in the moving average for better handing of the endpoints.

For the original differential sensor in Fig. 2 (c), the measured data form the sensor output and the input signal along with their moving averages are shown Fig. 10. The close of views indicate that the calculated moving average has produced almost noise free signals with respect to the collected data. Figure 11 shows the calculated noise component of the differential sensor output along with that of the measured input signal. A signal proportional to the actuation signal is also included in Fig. 11. Note that the noise level of the sensor output varies with the level of actuation. The noise level of the collected input signal is almost invariant with the actuation level, which confirms validity of the selected method for noise extraction. Using the standard deviation of the calculated sensor noise, the 


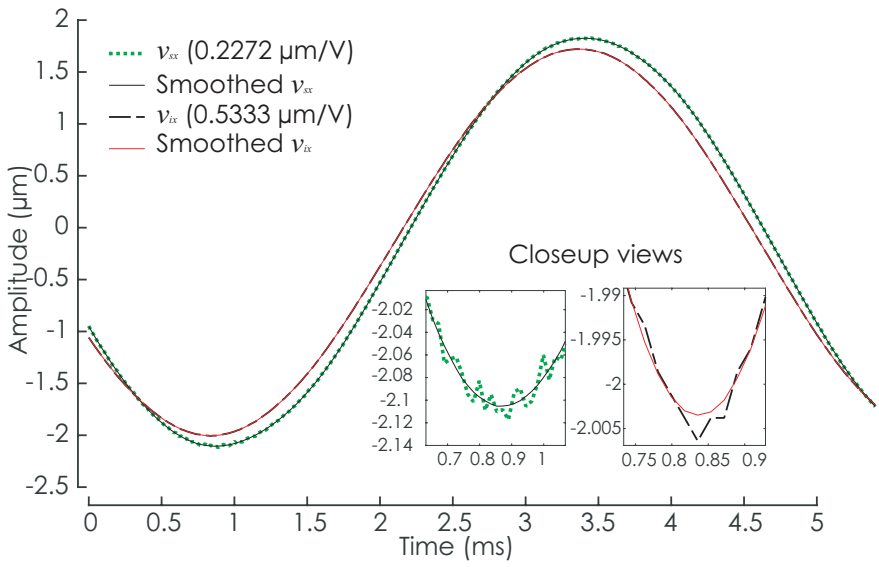

Figure 10. Differential sensor output of the readout circuit in Fig. 2 (c) and the input signal while driving the stage by a large signal sinusoidal excitation in the differential actuation mode. The data was collected by a sampling rate of $55.2 \mathrm{kHz}$, and the moving average span is 30 data points.

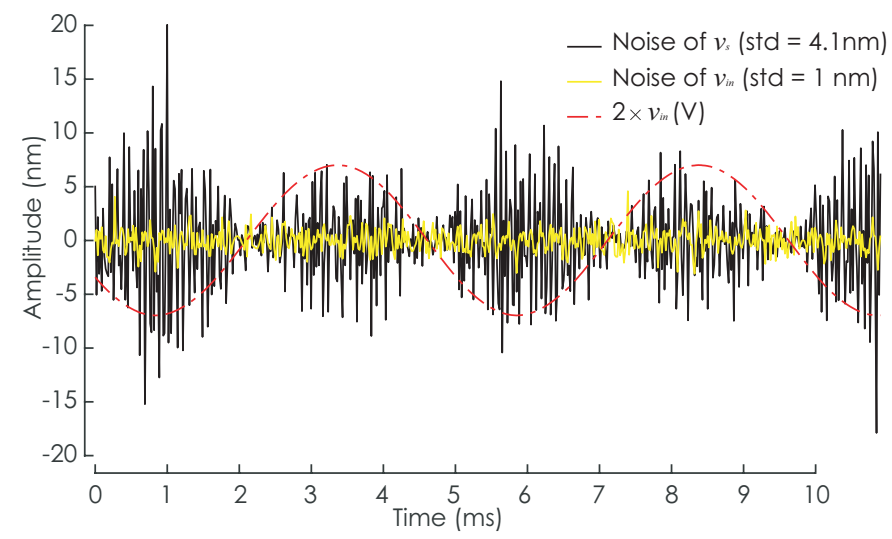

Figure 11. Calculated noise in the recorded input signal and differential sensor output of the readout circuit in Fig. 2 (c). Symbol "std" refers to standard deviation.

$3 \sigma$-resolution of the originally proposed differential sensor is $12.2 \mathrm{~nm}$.

To measure sensor noise for the differential sensor using the readout circuit in Fig. 3, we applied a low frequency large signal sinusoidal excitation to the actuators. The actuation input and sensor output signals along with their calculated moving averages are shown in Fig. 12 The resulting noise signals along with the scaled actuation input are depicted in Fig. 13. The noise level of the differential sensor again varies with the magnitude of input signal. The calculated $3 \sigma$-resolution of the sensor output is $12.9 \mathrm{~nm}$.

\section{Conclusions}

Differential actuation of piezo-driven nanopositioner provides bilateral motion for the stage and improved displacement range compared to the non-differential actuation modes. During the differential actuation, application of a differential sensing strategy can provide $62 \%$ improvement in the average measurement accuracy with respect to the non-differential sensing method $\left(\frac{191-71.8}{191}\right.$

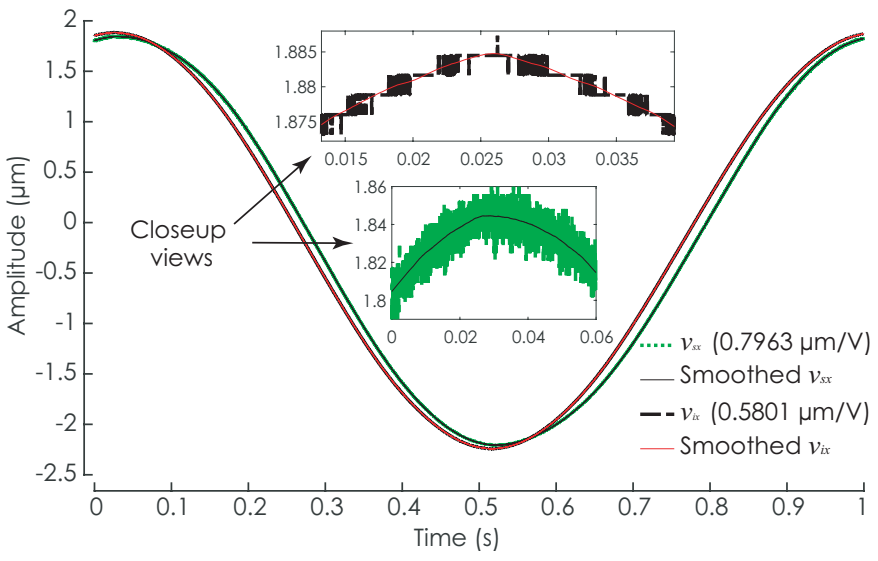

Figure 12. Differential sensor output of the readout circuit in Fig. 3 and the input signal while driving the stage by a large signal sinusoidal excitation in the differential actuation mode. The data was collected by a sampling rate of $70 \mathrm{kHz}$, and the moving average span is 600 data points.

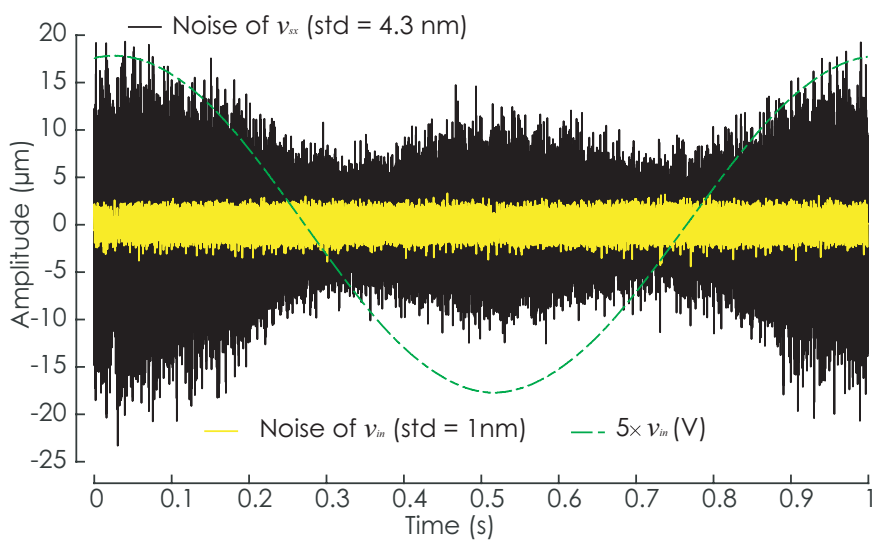

Figure 13. Calculated noise in the recorded input signal and differential sensor output of the readout circuit in Fig. 3 Symbol "std" refers to standard deviation.

from Fig. 6(b)). The proposed differential sensors employed commercial semiconductor strain gauges that are not only very small in size but also have large gauge factors. This resulted in a sensitivity of $\frac{1}{0.2272}=4.4 \frac{\mathrm{V}}{\mu \mathrm{m}}$, $3 \sigma$-resolution of $12.2 \mathrm{~nm}$, and bandwidth of $4 \mathrm{kHz}$ for the first proposed differential sensor in Fig. 2 (c). The second proposed differential sensing configuration in Fig. 3 has a smaller sensitivity of $\frac{1}{0.7963}=1.3 \frac{\mathrm{V}}{\mu \mathrm{m}}$ and a $3 \sigma$-resolution of $12.9 \mathrm{~nm}$. However, it is more compact and provides more accurate displacement sensing. Both differential sensors exhibit considerable variation of noise level as magnitude of input signal to differential actuation increases. Table III summarizes the main the experimental results for both differential sensors.

The stage has been tested in atmospheric conditions only. In vacuum conditions, we expect to see a slightly larger resonance frequency with a larger Q factor, due to a large reduction of air resistance. The sensor resolution may degrade due to lack of convective heat transfer between the piezoresistive strain gauges and ambient environment and larger thermal noise. The lack of convection may 
Table II

SUMMARIZED CHARACTERIZATION OF SENSOR OUTPUTS IN THE FIRST AND SECOND REAdOUT CIRCUITS IN Figures 2 (C) AND 3 Respectively. The abbreviations "Diff Out", "NondifF", "S.F.", "Calib.", "Valid.", "Res.", and "Sens.", refer to "Differential Output", "Non-differential Output", "Scale Factor", "Calibration Test", "Validation Test", "Resolution", and "Sensitivity", Respectively.

\begin{tabular}{ccccc}
\hline $\begin{array}{c}\text { Physical } \\
\text { Attribute }\end{array}$ & $\begin{array}{c}\text { Sensor 2 } \\
\text { Diff Out }\end{array}$ & $\begin{array}{c}\text { Sensor 1 } \\
\text { Diff Out }\end{array}$ & $\begin{array}{c}\text { Sensor 1 } \\
\text { Nondiff } v_{s x}^{+}\end{array}$ & $\begin{array}{c}\text { Sensor 1 } \\
\text { Nondiff } v_{s x}^{-}\end{array}$ \\
\hline S.F. $\left(\frac{\mu \mathrm{m}}{\mathrm{V}}\right)$ & 0.8 & 0.23 & 1.1513 & -1.104 \\
Calib. Error & $0.93 \%$ & $1.52 \%$ & $3.52 \%$ & $2.34 \%$ \\
Valid. Error & $1.05 \%$ & $1.96 \%$ & $5.2 \%$ & $2.9 \%$ \\
Res. $(\mathrm{nm})$ & 12.9 & 12.2 & 13.6 & 11.9 \\
Sens. $\left(\frac{\mathrm{V}}{\mu \mathrm{m}}\right)$ & 1.3 & 4.4 & 0.87 & -0.91 \\
\hline
\end{tabular}

also restrict the operating speed of the nanopositioner during large signal actuation. This is due to hysteresis nonlinearity, which determines the energy dissipated in the piezo stack in each cycle.

\section{Acknowledgment}

This work has been partially sponsored by the project PolyREM (Emergence Sorbonne Université), the French government research program Investissements d'avenir through the Robotex Equipment of Excellence (ANR-10EQPX-44), and the University of Newcastle Australia. We would like to thank Mr Ahmad Awde for providing recent photographs of the proposed device during the COVID-19 pandemic.

\section{REFERENCES}

[1] G. Schitter, K. J. Astrom, B. E. DeMartini, P. J. Thurner, K. L. Turner, and P. K. Hansma, "Design and modeling of a highspeed AFM-scanner," IEEE Transactions on Control Systems Technology, vol. 15, pp. 906-915, Sept 2007.

[2] E. Guliyev, B. E. Volland, Y. Sarov, T. Ivanov, M. Klukowski, E. Manske, and I. W. Rangelow, "Quasi-monolithic integration of silicon-MEMS with piezoelectric actuators for high-speed non-contact atomic force microscopy," Measurement Science and Technology, vol. 23, no. 7, p. 074012, 2012.

[3] D. E. Heverly, K.-W. Wang, and E. C. Smith, "Dual-stack piezoelectric device with bidirectional actuation and improved performance," Journal of Intelligent Material Systems and Structures, vol. 15, pp. 565-574, 072004.

[4] S. S. Aphale, A. J. Fleming, and S. O. R. Moheimani, "High speed nano-scale positioning using a piezoelectric tube actuator with active shunt control," IET Micro $\&$ Nano Letters, vol. 2, no. 1, pp. 9-12, 2007.

[5] S. Polit and J. Dong, "Development of a highbandwidth XY nanopositioning stage for high-rate micro/nanomanufacturing," IEEE/ASME Transactions on Mechatronics, vol. 16, pp. 724-733, Aug 2011.

[6] C.-X. Li, G.-Y. Gu, M.-J. Yang, and L.-M. Zhu, "Design, analysis and testing of a parallel-kinematic high-bandwidth XY nanopositioning stage," Review of Scientific Instruments, vol. 84, no. 12, p. 125111, 2013.

[7] M. Altaher and S. S. Aphale, "High-precision control of a piezodriven nanopositioner using fuzzy logic controllers," Computers, vol. 7, no. 1, p. Article No. 10, 2018

[8] M. R. Nabavi and S. Nihtianov, "A survey of eddy current displacement sensors: Imperfections and signal conditioning methods," in 16th International Scientific and Applied Science Conference, Book 3: Electronics ET 2007, (Sozopol, BULGARIA), pp. 116-122, Sept. 2007. ISSN: 1313-1842.
[9] M. R. Nabavi and S. N. Nihtianov, "Design strategies for eddycurrent displacement sensor systems: Review and recommendations," IEEE Sensors Journal, vol. 12, pp. 3346-3355, Dec 2012.

[10] A. J. Fleming, "A review of nanometer resolution position sensors: operation and performance," Sensors and Actuators A: Physical, vol. 190, pp. 106-126, 2013

[11] nPoint, Inc., https://www.npoint.com/npoint-products/, 1997.

[12] Mad City Labs Inc., PicoQ Sensor Technology, http://www.madcitylabs.com/picoq.html, 1999.

[13] W. O'Brien, "Piezoresistive sensors facilitate high-speed nanopositioning," Photonics Spectra, July 2003.

[14] H. Huang, H. Zhao, Z. Yang, Z. Fan, S. Wan, C. Shi, and Z. Ma, "Design and analysis of a compact precision positioning platform integrating strain gauges and the piezoactuator," Sensors, vol. 12, pp. 9697-9710, July 2012.

[15] Q. Xu, Deisign and Implementation of Large-Range Compliant Micropositioning Systems. John Wiley \& Sons, Singapore, Chapters 9 and 10, 2016.

[16] T.-P. Dao and S.-C. Huang, "Design and analysis of a compliant micro-positioning platform with embedded strain gauges and viscoelastic damper," Microsystem Technologies, vol. 23, pp. 441-456, Feb 2017.

[17] A. Furuta, M. Munekata, and T. Higuchi, "Precise positioning stage driven by multilayer piezo actuator using strain gauge," Japanese Journal of Applied Physics, vol. 41, pp. 6283-6286, Oct. 2002

[18] N. Hosseini, A. P. Nievergelt, J. D. Adams, V. T. Stavrov, and G. E. Fantner, "A monolithic MEMS position sensor for closed-loop high-speed atomic force microscopy," Nanotechnology, vol. 27, p. 135705, feb 2016.

[19] A. J. Fleming and K. K. Leang, "Integrated strain and force feedback for high performance control of piezoelectric actuators," Sensors and Actuators A, vol. 161, no. 1-2, pp. 256-265, 2010.

[20] Y. K. Yong and A. J. Fleming, "High-speed vertical positioning stage with integrated dual-sensor arrangement," Sensors and Actuators A: Physical, vol. 248, pp. $184-192,2016$.

[21] A. Bazaei, M. Boudaoud, M. H. Ettefagh, Z. Chen, and S. Régnier, "In-situ semiconductor position sensor for differentially piezo-driven nanopositioners," in 2019 IEEE SENSORS Conference, pp. 1-4, Oct 2019.

[22] A. Bazaei, M. Boudaoud, M. H. Ettefagh, Z. Chen, and S. Régnier, "Displacement sensing by piezoelectric transducers in highspeed lateral nanopositioning," IEEE Sensors Journal, vol. 19 pp. 9156-9165, Oct. 2019.

[23] Thorlabs Inc., https://www.thorlabs.com/navigation.cfm?guide $i d=82,1989$.

[24] Tianming Lu, M. Boudaoud, D. Hériban, and S. Régnier, "Nonlinear modeling for a class of nano-robotic systems using piezoelectric stick-slip actuators," in 2015 IEEE/RSJ International Conference on Intelligent Robots and Systems (IROS), pp. 6020-6025, Sept. 2015.

[25] S. Liang, M. Boudaoud, B. Cagneau, and S. Régnier, "Velocity characterization and control strategies for nano-robotic systems based on piezoelectric stick-slip actuators," in 2017 IEEE International Conference on Robotics and Automation (ICRA), pp. 6606-6611, June 2017.

[26] M. Boudaoud, T. Lu, S. Liang, R. Oubellil, and S. Régnier, "A voltage/frequency modeling for a multi-dofs serial nanorobotic system based on piezoelectric inertial actuators," IEEE/ASME Transactions on Mechatronics, vol. 23, no. 6, pp. 2814-2824, 2018.

[27] R. Oubellil, A. Voda, M. Boudaoud, and S. Régnier, "Mixed stepping/scanning mode control of stick-slip sem-integrated nano-robotic systems," Sensors and Actuators A: Physical, vol. 285 , pp. $258-268$, Jan. 2019.

[28] Mad City Labs Inc., Nano-HS2 Nanopositioner, http://www.madcitylabs.com/nanohsseries.html, 1998.

[29] PI (Physik Instrumente), P-363 PicoCube XY Piezo Scanner, https://www.pi-usa.us/en/products/piezo-flexurenanopositioners/xyz-piezo-flexure-nanopositioning-stages $/ p$ 363-picocube-xyz-piezo-scanner-201650/\#specification, 1994.

[30] PIEZOCONCEPT Inc., LFHS2 nanopositioner, http://www.piezoconcept.com/products-2-axis-stages/lfhs-2-2/, 2013. 
[31] PIEZOCONCEPT Inc., LTHS2 stage, http://www.piezoconcept.com/products-2-axis-stages/lths2/, 2013.

[32] PI (Physik Instrumente), P-541.2DD XY Piezo Stage, https://www.physikinstrumente.com/en/products/nanoposition ing-piezo-flexure-stages/xy-piezo-flexure-stages /p-5412-p5422-xy-piezo-stage-201530/\#specification, 1994.

[33] Micron Instruments, https://www.microninstruments.com/cate gory/strain-gage-solutions, 1979.

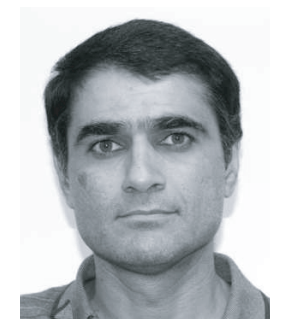

Ali Bazaei (M'10) received B.Sc. and M.Sc. degrees from Shiraz University and a Ph.D. degree from Western University, all in electrical engineering in 1992, 1995, and 2009, respectively. Since 2009, he held postdoctoral research fellowship, research associate, and higher degree research positions with University of Newcastle, Australia and with Institute for Intelligent Systems and Robotics (ISIR) at Sorbonne University. His research interests include the general area of nonlinear systems including control and modeling of structurally flexible systems, friction modeling and compensation, neural networks, and micro-position sensors, and Nano-positioning in Scanning Probe Microscopy.

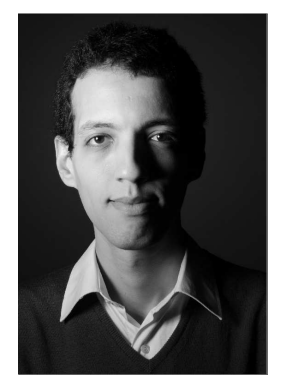

Mokrane Boudaoud received the engineering degree in automatic control from the University of science and technology Houari Boumédiène (USTHB, Algiers, Algeria), and a MS degree in mechatronics and microrobotics at the University of Franche-Comté (UFC, Besançon, France) in 2009. He received in 2012 the $\mathrm{PhD}$ degree in control engineering at AS2M (Automatic Control and MicroMechatronic Systems) department of Femto-st Institute. He is currently Associate Professor at University Pierre et Marie Curie / Institut des Systèmes Intelligents et de Robotique. His field of interest includes modeling and robust control of multi-degree-of-freedom nano-robotic systems operating under a scanning electron microscope (SEM). Dr. Boudaoud is an Associate Editor of the journal Mechatronics (Elsevier).

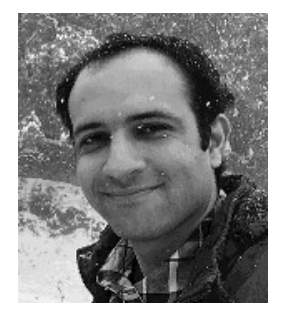

Massoud Hemmasian Ettefagh received his B.Sc. degree from Isfahan University of Technology, Iran in 2008, and M.Sc. and P.h.D. degrees from Amirkabir University of Technology, Iran in 2011 and 2018, all in mechanical engineering. During 2016-2019 he was a visiting fellow at University of Newcastle, Australia and Universidad Carlos III de Madrid, Spain. Currently, he is with the Huazhong University of Science and Technology (HUST), China as a postdoctoral fellowship. His research interests include real-time control, constrained control, model predictive control, time-varying systems, differential geometric methods in nonlinear systems, and design and control of nanopositioning platforms for AFMs.

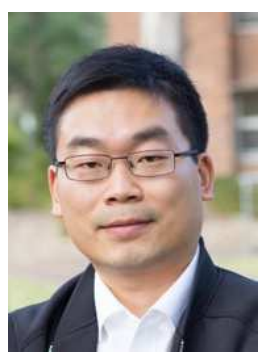

Zhiyong CHEN received the B.E. degree from the University of Science and Technology of China, and the M.Phil. and Ph.D. degrees from the Chinese University of Hong Kong, in 2000, 2002 and 2005, respectively. $\mathrm{He}$ worked as a research associate at the University of Virginia during 2005-2006. He joined the University of Newcastle, Australia, in 2006, where he is currently a professor. He is also a Changjiang chair professor with Central South University, Changsha, China. His research interests include non-linear systems and control, biological systems, and multi-agent systems. He is/was an associate editor of Automatica, IEEE Transactions on Automatic Control and IEEE Transactions on Cybernetics.

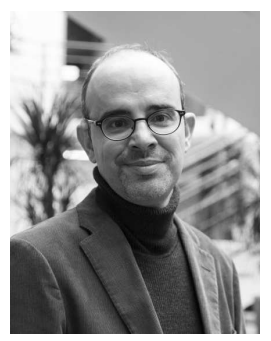

Stéphane Régnier received his $\mathrm{PhD}$ degree in Mechanics and Robotics from the University of Pierre and Marie Curie, Paris, France in 1996. He is currently Professor at the Institute of Intelligent Systems and Robotics (ISIR), University of Pierre and Marie Curie, Paris, France. He has been head of the ISIR micromanipulation team since 2001. His research interests are focused on micro and nano manipulation, teleoperation and haptic feedback logical cell characterization. 